\title{
Determination of Ground- and Excited-State Dipole Moments of Pyronin B Using the Solvatochromic Method and Quantum-Chemical Calculations
}

\author{
B. ACEMIOĞLU ${ }^{a, *}$ AND Y. ONGANER ${ }^{b}$ \\ ${ }^{a}$ Department of Chemistry, Faculty of Science and Arts, \\ Kilis 7 Aralik University, 79000, Kilis, Turkey \\ ${ }^{b}$ Department of Chemistry, Faculty of Science and Arts, Atatürk University, \\ 25240, Erzurum, Turkey
}

Received: 14.04.2020 \& Accepted: 25.05.2020

Doi: 10.12693/APhysPolA.138.546

*e-mail: acemioglu@kilis.edu.tr

\begin{abstract}
Ground- and excited-state dipole moments of pyronin B (PyB) in n-alcohols (methanol to n-hexanol), acetonitrile and acetonitrile-benzene solvent mixtures with different polarity were determined using the solvatochromic method and quantum-chemical calculations. The ground-state dipole moments were calculated using both the experimental Guggenheim-Smith method (GSM) and the theoretical (SCF MO LCAO) in CNDO/2 and MNDO methods. The values of the theoretical dipole moments of PyB were determined to be 11.29 and $3.43 \mathrm{D}$ (Debye) for the CNDO/2 and MNDO methods, respectively. Experimental ground-state dipole moments for PyB increased from 3.13 to $13.77 \mathrm{D}$ in n-alcohols, and 9.25 to 14.63 in $\mathrm{AN}$ and $\mathrm{AN}-\mathrm{BN}$ solvent mixtures, with decreasing polarity. The excited-state dipole moment values were determined from Lippert's and Bakhshiev's equations. The ground-state dipole moments were found to be higher than the excited-state moments. It was determined that the groundstate structure was more polar and stable as compared to the excited-state
\end{abstract}

topics: pyronin B, solvatochromic shift, ground- and excited-state dipole moments, solute-solvent interactions

\section{Introduction}

The solvatochromic method is based on the spectral shift of absorption and fluorescence maxima with solvent effects. This method has also been used to determine the excited-state dipole moments of molecules [1-7]. The electronic absorption and emission spectra of organic molecules change as a function of solvent polarity. The difference between the wavenumbers of maxima of the absorption and emission spectra is the Stokes shift [3]. Upon excitation, the excited-state dipole moments of the molecules have been determined using the solvent-dependent Stokes shifts and the solvent polarity functions which are a function of the dielectric constant and refractive index by Lippert's, Bakhshiev's and Chamma-Viallet's equations [4-6]. The plots of the Stokes shift against solvent polarity functions using Lippert's, Bakhshiev's and Chamma-Viallet's equations should be linear in the case of general solvent interactions [4]. These equations do not consider specific solute-solvent interactions such as a hydrogen bonding effect and complex formation or charged states of excited states and the occurring deviations from the linearity of plots of the Stokes shift $[3,6]$.
On the other hand, the ground-state dipole moments of the molecules can be determined using both theoretical and experimental methods. As a theoretical method, the quantumchemical calculations have frequently been used to determine the ground-state dipole moments of the molecules $[7,8]$. As an experimental method, the Guggenheim-Smith method (GSM) [9] has been used. Our aim was to determine the ground- and excited-state dipole moments of pyronin $\mathrm{B}$ (PyB) which is a xanthene dye, and has not been studied in the literature so far. It is well known that PyB is an environment-sensitive dye, widely used in biological and synthetic polyelectrolyte systems [10], aside from its use in the laser industry [11]. Such dyes are greatly affected by environmental factors including solvent polarity, concentration, $\mathrm{pH}$, etc. [12]. These environmental factors cause spectral shifts by affecting the absorption and fluorescence spectra of a dye molecule. In this study, the ground- and excitedstate dipole moments of $\mathrm{PyB}$ were determined in polar protic and polar aprotic solvents using theoretical and solvatochromism methods. We used $\mathrm{n}$-alcohols (methanol to $\mathrm{n}$-haxanol) as polar protic solvents, and acetonitrile and acetonitrile-benzene solvent mixtures as polar aprotic solvents. 


\section{Experimental}

PyB was obtained from Sigma-Aldrich and used as received without any purification. The chemical structure of $\mathrm{PyB}$ is shown in Fig. 1. The n-alcohol solvents (methanol to n-hexanol), and acetonitrile $(\mathrm{AN})$ and benzene $(\mathrm{BN})$ solvents, obtained from Merck, were of spectroscopic grade. The n-alcohols were used by distilling over $\mathrm{CaH}_{2}$. $\mathrm{AN}$ and $\mathrm{BN}$ solvents were used as received. Stock solution of $1.0 \mathrm{mM}$ of PyB was prepared in methanol and then used freshly in the experiments. For the experiments, the sample of $5 \mu \mathrm{l}$ was taken from the stock solution and then evaporated by argon gas. Then, $5 \mathrm{ml}$ of n-alcohols, $\mathrm{AN}$ and AN-BN solvent mixtures were added to the evaporated samples, respectively. The final concentrations prepared were $1 \times 10^{-6} \mathrm{M}$ in $\mathrm{n}$-alcohols and the mixtures. Absorption spectra were recorded using a Shimadzu UV-VIS 160A spectrophotometer at $1.0 \mathrm{~nm}$ slit width. Fluorescence spectra were recorded at $5 \mathrm{~nm}$ and $1.5 \mathrm{~nm}$ slit widths for excitation and emission at $90^{\circ}$ of the incident excitation beam using a Shimadzu RF-5301 PC spectrofluorophotometer. Jacobian transformation states of the obtained absorption and fluorescence spectra of $\mathrm{PyB}$ in n-alcohols, $\mathrm{AN}$ and $\mathrm{AN}-\mathrm{BN}$ solvent mixtures are shown in Figs. 2 and 3. The capacitance values (the values of $C$ and $C_{0}$ ) for the dielectric constants $(D)$ were measured using a capacitor by a LCR meter (Hewlett Packard 4284 A $20 \mathrm{~Hz}-1 \mathrm{MHz}$ Precision LCR meter). The refraction index $(n)$ was determined using a refractometer (Jena). All measurements were recorded at room temperature of $25^{\circ} \mathrm{C}$.

\section{Results and discussion}

\subsection{Ground-state dipole moments}

The ground-state dipole moments of $\mathrm{PyB}$ were determined using both theoretical and experimental methods. The theoretical dipole moments were determined with the SCF MO LCAO in the CNDO $/ 2$ and MNDO methods [12]. The experimental ground-state dipole moments were calculated in n-alcohols, $\mathrm{AN}$ and $\mathrm{AN}-\mathrm{BN}$ solvent mixtures according to the GSM equation [9], namely:

$$
\mu_{g}=\frac{27 k_{\mathrm{B}} T}{4 \pi d} \frac{A_{D}-A_{n}}{(D+2)^{2}} M,
$$

where $k_{\mathrm{B}}$ is the Boltzmann constant, $T$ is the absolute temperature, $d$ is the density of the solvent, $D$ is its dielectric constant, and $M$ is the molecular<smiles>CCN(CC)c1ccc2cc3ccc(=[N+](CC)CC)cc-3oc2c1</smiles>

Fig. 1. The chemical structure of pyronin B.

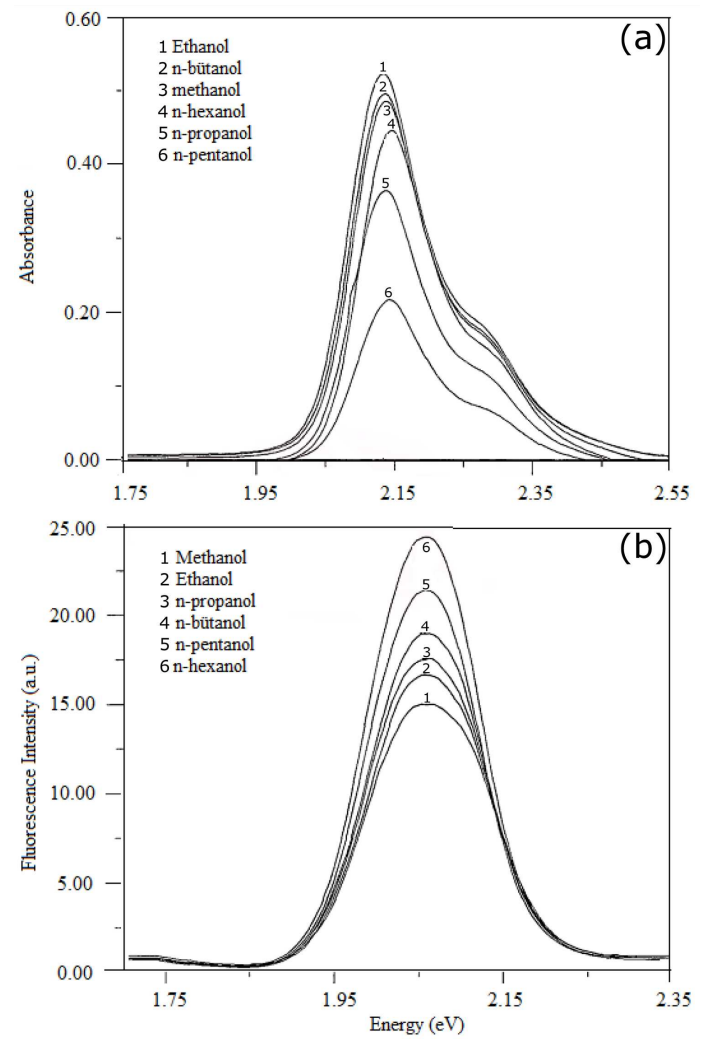

Fig. 2. Spectra of PyB in n-alcohols: (a) Absorption spectra, (b) Fluorescence spectra.

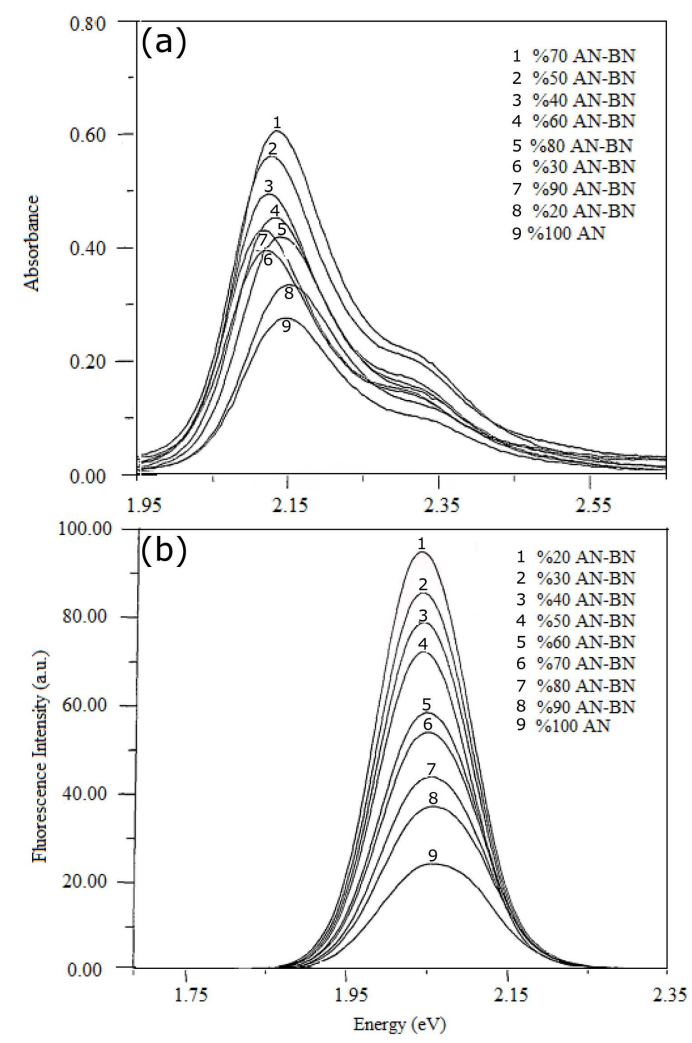

Fig. 3. Spectra of $\mathrm{PyB}$ in $\mathrm{AN}$ and $\mathrm{AN}-\mathrm{BN}$ solvent mixtures: (a) Absorption spectra, (b) Fluorescence spectra. 
Dipole moments of PyB in the ground states (in Debye).

TABLE I

\begin{tabular}{|c|c|c|c|}
\hline Solvent & $\mu_{e}$ & $\overline{\Delta \Delta \mu}$ & $\mu_{g}^{\text {Theor }}$ \\
\hline \multirow{4}{*}{ n-alcohols } & $1.61(\mathrm{MNDO})$ & \multirow{2}{*}{1.82 (from Lippert Eq.) } & \multirow{8}{*}{$\begin{array}{l}3.43 \text { (from MNDO) } \\
11.29(\text { from } \mathrm{CNDO} / 2)\end{array}$} \\
\hline & $9.47(\mathrm{CNDO} / 2)$ & & \\
\hline & $2.30(\mathrm{MNDO})$ & \multirow{2}{*}{1.13 (from Bakhshiev Eq.) } & \\
\hline & $10.16(\mathrm{CNDO} / 2)$ & & \\
\hline \multirow{4}{*}{ AN-BN solvent mixture } & $0.93(\mathrm{MNDO})$ & \multirow{2}{*}{2.50 (from Lippert Eq.) } & \\
\hline & $8.79(\mathrm{CNDO} / 2)$ & & \\
\hline & $1.94(\mathrm{MNDO})$ & \multirow{2}{*}{1.49 (from Bakhshiev Eq.) } & \\
\hline & $9.80(\mathrm{CNDO} / 2)$ & & \\
\hline
\end{tabular}

TABLE II

The experimental ground-state dipole moments calculated from the GSM ( $\left.\mu_{g}^{\text {exper }}\right)$ and excited-state dipole moments $\left(\mu_{e}\right)$ calculated with (2) and (3) of $\mathrm{PyB}$ in the solvents. AN-BN reads as volume percentages of acetonitrile to benzene.

\begin{tabular}{l|c|c|c}
\hline \hline \multirow{2}{*}{ Solvent } & \multirow{2}{*}{$\mu_{g}^{\text {exper }}$} & \multicolumn{2}{|c}{$\mu_{e}$} \\
\cline { 3 - 4 } & & $\begin{array}{c}\text { Lippert's } \\
\text { Eq. (2) }\end{array}$ & $\begin{array}{c}\text { Bakhshiev's } \\
\text { Eq. (3) }\end{array}$ \\
\hline Methanol & 3.13 & 1.31 & 2.92 \\
Ethanol & 4.95 & 3.13 & 4.81 \\
n-propanol & 8.56 & 6.74 & 8.48 \\
n-butanol & 10.19 & 8.37 & 10.12 \\
n-pentanol & 12.17 & 11.15 & 12.92 \\
n-hexanol & 13.77 & 11.95 & 13.72 \\
Acetonitrile & 9.25 & 6.75 & 9.12 \\
90\%AN-10\%BN & 9.81 & 7.31 & 9.69 \\
80\%AN-20\%BN & 10.04 & 7.54 & 9.93 \\
70\%AN-30\%BN & 10.34 & 7.84 & 10.23 \\
$60 \%$ AN-40\%BN & 10.71 & 8.21 & 10.60 \\
50\%AN-50\%BN & 11.19 & 8.69 & 11.09 \\
40\%AN-60\%BN & 11.47 & 8.97 & 11.37 \\
30\%AN-70\%BN & 13.26 & 10.77 & 13.17 \\
20\%AN-80\%BN & 14.63 & 12.17 & 14.55
\end{tabular}

weight of the solute dye. Further, $A_{D}$ and $A_{n}$ are the numerical values obtained from the solution dielectric constant and refractive index measurements, respectively. The used dielectric constants of the solvent and solutions were determined as follows: $D=C / C_{0}$, where $C$ and $C_{0}$ are capacitances which are measured when the capacitor is full or empty with solvent or solution, respectively.

The values of the theoretical dipole moments $\mu_{g}^{\text {theor }}$ of PyB were calculated as 11.29 D (Debye) for $\mathrm{CNDO} / 2$ and $3.43 \mathrm{D}$ for MNDO. The value of $\mu_{q}^{\text {theor }}$ obtained from CNDO/2 was 3.29 times higher than that of MNDO (see Table I). It was seen that the values of $\mu_{g}^{\text {theor }}$ obtained from CNDO $/ 2$ were in agreement with the values of the experimental ground-state dipole moments $\mu_{g}^{\text {exper }}$ estimated from the GSM in n-alcohols and AN-BN solvent mixtures (see Table II).
According to the GSM method, the experimental ground-state dipole moments $\mu_{g}^{\text {exper }}$ of PyB were determined in $\mathrm{n}$-alcohols, and in $\mathrm{AN}$ and $\mathrm{AN}-\mathrm{BN}$ solvent mixtures (see Table II). From Table II, it is seen that $\mu_{g}^{\text {exper }}$ values of PyB increase with the decrease in the solvent polarity in a series of n-alcohols (methanol to n-hexanol) and in AN and AN-BN solvent mixtures. For example, it was determined that $\mu_{g}^{\text {exper }}$ values of $\mathrm{PyB}$ increased from 3.13 to 13.77 in a series of n-alcohols and grew from 9.25 to 14.63 in $\mathrm{AN}$ and $\mathrm{AN}-\mathrm{BN}$ solvent mixtures, with the decreasing values of polarity.

\subsection{Excited-state dipole moments}

The experimental excited-state dipole moments of $\mathrm{PyB}$ in n-alcohols and $\mathrm{AN}$ and $\mathrm{AN}-\mathrm{BN}$ solvent mixtures were determined according to Lippert's, Bakhshiev's and Chamma-Viallet's equations.

Lippert's equation [13] can be expressed as

$$
\bar{\nu}_{a}-\bar{\nu}_{f}=\frac{2 \Delta \mu^{2}}{a_{0}^{3} h c}\left(\frac{D-1}{2 D+1}-\frac{n^{2}-1}{2 n^{2}+1}\right),
$$

where $\Delta \mu^{2}=\left(\mu_{e}-\mu_{g}\right)^{2}$, and $\mu_{g}$ and $\mu_{e}$ refer to the dipole moments of the ground- and excitedstates, respectively. The absorption and emission wavenumbers at maxima, respectively, are denoted as $\bar{\nu}_{a}$ and $\bar{\nu}_{f}\left(\mathrm{~cm}^{-1}\right)$, the cavity radius of the solute is denoted as $a_{0}$, and $D$ and $n$ are the dielectric constant and the refractive index of the solvent, respectively.

Bakhshiev's equation [14] reads as

$$
\bar{\nu}_{a}-\bar{\nu}_{f}=\frac{2 \Delta \mu^{2}}{a_{0}^{3} h c}\left[\frac{D-1}{D+2}-\frac{n^{2}-1}{n^{2}+2}\right] \frac{\left(2 n^{2}+1\right)}{\left(n^{2}+2\right)},
$$

Chamma-Viallet's equation [15] is given as

$$
\begin{aligned}
& \frac{\bar{\nu}_{a}+\bar{\nu}_{f}}{2}=-\frac{2 \Delta \mu^{2}}{a_{0}^{3} h c} \\
& \times\left[\frac{2 n^{2}+1}{2\left(n^{2}+2\right)}\left(\frac{D-1}{D+2}-\frac{n^{2}-1}{n^{2}+2}\right)-\frac{3\left(n^{4}-1\right)}{2\left(n^{2}+2\right)^{2}}\right],
\end{aligned}
$$

where $\Delta \mu^{2}$ is equal to $\left(\mu_{e}^{2}-\mu_{g}^{2}\right)$, the remaining symbols in (3) and (4) are the same as in (2). The cavity radius of the solute molecule is $a_{0}$ and it is determined in the following way [16]:

$$
a_{0}=\left(\frac{3 M}{4 \pi \delta N_{\mathrm{A}}}\right)^{1 / 3},
$$


TABLE III

Some physical properties: $F_{1}, F_{2}, F_{3}$ - solvent polarity function calculated with (6), (7) and (8), respectively; $n_{D}^{25}$ - refractive index of the solvent; $D^{25}$ — dielectric constant of the solvent; $E_{T}(30)$ - solvent polarity parameters from Ref. [4]. AN-BN reads as volume percentages of acetonitrile to benzene.

\begin{tabular}{l|c|c|c|c|c|c}
\hline \hline \multicolumn{1}{c|}{ Solvent } & $F_{1}$ & $F_{2}$ & $F_{3}$ & $n_{D}^{25}$ & $D^{25}$ & $E_{T}(30)$ \\
\hline methanol & 0.309 & 0.854 & 0.651 & 1.3280 & 32.58 & 55.13 \\
ethanol & 0.290 & 0.813 & 0.649 & 1.3602 & 24.64 & 52.00 \\
n-propanol & 0.275 & 0.779 & 0.650 & 1.3833 & 20.15 & 50.35 \\
n-butanol & 0.264 & 0.751 & 0.645 & 1.3974 & 17.44 & 49.53 \\
n-pentanol & 0.250 & 0.703 & 0.627 & 1.4064 & 14.00 & 49.12 \\
n-hexanol & 0.245 & 0.692 & 0.629 & 1.4165 & 13.30 & 48.60 \\
acetonitrile & 0.307 & 0.861 & 0.663 & 1.3420 & 37.12 & 45.41 \\
$90 \% \mathrm{AN}-10 \% \mathrm{BN} *$ & 0.301 & 0.852 & 0.665 & 1.3521 & 34.41 & 44.31 \\
80\%AN-20\%BN & 0.294 & 0.836 & 0.666 & 1.3642 & 30.46 & 44.00 \\
$70 \% \mathrm{AN}-30 \% \mathrm{BN}$ & 0.287 & 0.817 & 0.662 & 1.3722 & 26.06 & 43.60 \\
$60 \% \mathrm{AN}-40 \% \mathrm{BN}$ & 0.279 & 0.802 & 0.666 & 1.3903 & 24.30 & 43.00 \\
$50 \% \mathrm{AN}-50 \% \mathrm{BN}$ & 0.270 & 0.785 & 0.667 & 1.4044 & 22.17 & 42.40 \\
$40 \% \mathrm{AN}-60 \% \mathrm{BN}$ & 0.249 & 0.715 & 0.645 & 1.4245 & 15.18 & 42.00 \\
$30 \% \mathrm{AN}-70 \% \mathrm{BN}$ & 0.233 & 0.665 & 0.628 & 1.4427 & 12.36 & 40.80 \\
$20 \% \mathrm{AN}-80 \% \mathrm{BN}$ & 0.218 & 0.652 & 0.626 & 1.4616 & 10.67 & 39.96
\end{tabular}

where $\delta$ is the density of the solute molecule, $M$ is the molecular weight of the solute and $N_{\mathrm{A}}$ is Avogadro's number. Herein, we determined $a_{0}=5.28 \AA$ for PyB.

From (2-4), solvent polarity functions depending on the dielectric constant and refractive index, can be expressed with $F_{1}(D, n), F_{2}(D, n)$ and $F_{3}(D, n)$ symbols.

$$
\begin{aligned}
& F_{1}(D, n)=\frac{D-1}{2 D+1}-\frac{n^{2}-1}{2 n^{2}+1}, \\
& F_{2}(D, n)=\frac{2 n^{2}+1}{n^{2}+2}\left(\frac{D-1}{D+2}-\frac{n^{2}-1}{n^{2}+2}\right), \\
& F_{3}(D, n)=\frac{F_{2}(D, n)}{2}+\frac{3\left(n^{4}-1\right)}{2\left(n^{2}+2\right)^{2}} .
\end{aligned}
$$

The values of $F_{1}(D, n), F_{2}(D, n), F_{3}(D, n)$ for n-alcohols, AN and AN-BN solvent mixtures are listed in Table III [4].

Solvent-dependent spectral data of $\mathrm{PyB}$ are given in Table IV. The maximum wavenumbers of the absorption and fluorescence emission spectra were used to determine the Stokes shifts in n-alcohols and mixtures. It was observed that the maxima of both the absorption and fluorescence emission spectra of PyB were blue-shifted slightly with increasing solvent polarity in n-alcohols and the mixtures (see Table IV). In order to determine the excited-state dipole moment values, the Stokes shifts $\left(\bar{\nu}_{a}-\bar{\nu}_{f}\right)$ and the arithmetic means of Stokes shifts $\left(\bar{\nu}_{a}+\bar{\nu}_{f}\right) / 2$ were plotted against the solvent polarity functions $\left(F_{1}, F_{2}\right.$, and $\left.F_{3}\right)$, according to (2)-(4). Lippert's, Bakhshiev's and ChammaViallet's plots are presented in Figs. 4-6. The characteristics of the correlations, i.e., the slopes, intercepts, and determination of coefficient $r^{2}$ values, were determined from the linear regression analyses of these plots. According to these three equations, the plots of the Stokes shifts versus solvent polarity functions should be linear in the presence of the general solvent effect. Deviations from linearity can occur in the presence of specific solutesolvent effects such as hydrogen bonding formation between the solute and the solvent $[3,4,17]$. These deviations are related to the extent of interactions between the solute and solvent molecules.

According to the relationship $\mu_{g}=\mu_{e}+\Delta \mu$ [17], due to the blue shift of PyB in all solvents, the values of excited-state dipole moments $\mu_{e}$ were calculated using the values of $\Delta \mu$ obtained from Lippert's and Bakhshiev's equations (except for ChammaViallet's equation) and the $\mu_{g}^{\text {theor }}$ values estimated from the MNDO and $\mathrm{CNDO} / 2$ approximations in n-alcohols and in the mixtures. According to Chamma-Viallet's equation, the plots of the Stokes shift versus the solvent polarity function $\left(F_{3}\right)$ did not show a harmony due to their positive slopes in n-alcohols and the mixtures (see Fig. 4), and therefore the excited-state dipole moments of PyB could not be determined from Chamma-Viallet's equation. The same results with a positive slope for Chamma-Viallet's equation have also been obtained for caffeine in various solvents [18] and fluorescein dye in acetonitrile and acetonitrile-benzene mixtures [4]. The values of $\mu_{g}^{\text {theor }}$ obtained from the MNDO and CNDO/2 approximations were found as 3.23 and $11.29 \mathrm{D}$, respectively. The values of $\mu_{e}$ were determined using $\mu_{g}^{\text {theor }}$ values and the values of $\Delta \mu$ were obtained from Lippert's and Bakhshiev's equations. By using $\Delta \mu$ obtained from Lippert's equation, the values of $\mu_{e}$ were determined as 1.61 D (MNDO) and 9.47 D (CNDO/2) in a series of n-alcohols, and also $0.93 \mathrm{D}$ (MNDO) 
TABLE IV

Spectral data of PyB in the solvents: wavelength of absorption maxima $\left(\lambda_{a}\right)$, wavelength of fluoresence emission maxima $\left(\lambda_{f}\right)$, wavenumber of absorption maxima $\left(\bar{\nu}_{a}\right)$, wavenumber of fluorescence emission maxima $\left(\bar{\nu}_{b}\right)$, Stokes shift $\left(\bar{\nu}_{a}-\bar{\nu}_{f}\right)$, mean of Stokes shift $\left(\left(\bar{\nu}_{a}+\bar{\nu}_{f}\right) / 2\right)$. AN-BN reads as volume percentages of acetonitrile to benzene.

\begin{tabular}{l|c|c|c|c|c|c}
\hline \multicolumn{1}{c|}{ Solvent } & $\lambda_{a}[\mathrm{~nm}]$ & $\lambda_{f}[\mathrm{~nm}]$ & $\bar{\nu}_{a}\left[\mathrm{~cm}^{-1}\right]$ & $\bar{\nu}_{f}\left[\mathrm{~cm}^{-1}\right]$ & $\left(\bar{\nu}_{a}-\bar{\nu}_{f}\right)\left[\mathrm{cm}^{-1}\right]$ & $\left(\bar{\nu}_{a}+\bar{\nu}_{f}\right) / 2\left[\mathrm{~cm}^{-1}\right]$ \\
\hline methanol & 551 & 575 & 18149 & 17391 & 758 & 17770 \\
ethanol & 552 & 576 & 18116 & 17361 & 755 & 17739 \\
n-propanol & 553 & 576 & 18083 & 17361 & 722 & 17722 \\
n-butanol & 553 & 576 & 18083 & 17361 & 722 & 17722 \\
n-pentanol & 554 & 576 & 18051 & 17361 & 690 & 17706 \\
n-hexanol & 555 & 578 & 18018 & 17301 & 717 & 17660 \\
acetonitrile & 553 & 576 & 18083 & 17361 & 722 & 17722 \\
$90 \%$ AN-10\%BN* & 554 & 576 & 18051 & 17361 & 690 & 17706 \\
$80 \%$ AN-30\%BN & 555 & 577 & 18018 & 17331 & 687 & 17675 \\
$70 \%$ AN-30\%BN & 556 & 577 & 17986 & 17331 & 655 & 17659 \\
$60 \%$ AN-40\%BN & 556 & 577 & 17986 & 17331 & 655 & 17659 \\
$50 \%$ AN-50\%BN & 557 & 578 & 17953 & 17301 & 652 & 17627 \\
$40 \% A N-60 \% B N$ & 558 & 578 & 17921 & 17301 & 620 & 17611 \\
$30 \% A N-70 \% B N$ & 559 & 578 & 17889 & 17301 & 588 & 17595 \\
$20 \% A N-80 \% B N$ & 560 & 578 & 17857 & 17301 & 556 & 17579
\end{tabular}

and $8.79 \mathrm{D}(\mathrm{CNDO} / 2)$ in $\mathrm{AN}$, and $\mathrm{AN}-\mathrm{BN}$ solvent mixtures, respectively. Likewise, by using $\Delta \mu$ obtained from Bakhshiev's equation, the values of $\mu_{e}$ were estimated as $2.30 \mathrm{D}(\mathrm{MNDO})$ and $10.16 \mathrm{D}$ $(\mathrm{CNDO} / 2)$ in a series of n-alcohols, and $1.94 \mathrm{D}$ (MNDO) and 9.80 D (CNDO/2) in AN and AN-BN solvent mixtures, respectively.

On the other hand, it was determined that the excited-state dipole moments $\mu_{e}$ from Lippert's and Bakhshiev's equations in n-alcohols and the mixtures were lower than the values of both $\mu_{g}^{\text {theor }}$ and $\mu_{g}^{\text {exper }}\left(\mu_{e}<\mu_{g}\right)$ (see Table II). The fact that $\mu_{g}$ is larger than $\mu_{e}$ is uncommon. This may result from the blue shift of absorption and fluorescence emission spectra with the increasing polarity function upon excitation. When the $\mathrm{PyB}$ molecule is excited by the effect of the incident excitation beam, it has been reported that an internal charge transfer in $\mathrm{PyB}$ does not occur in the excited-state [10]. In the excited-state, PyB showed the solute-solvent interactions via internal conversion only. Therefore, in this study, the values of the $\mu_{e}$ were found smaller than those of $\mu_{g}$. Similar results have also been reported for merocyanine 540 dye in nitrile solvents [17], fluorescein in n-alcohols and acetonitrile-benzene mixtures [4], and purine and purine derivatives in various solvents [18]. Since $\mu_{e}$ values are smaller than $\mu_{g}$ values, it may be said that the excitedstate of $\mathrm{PyB}$ is less polar relative to the groundstate [18]. At the same time, these results indicate a more stable ground-state structure as compared to the excited-state [19].

The values of determination of coefficient $r^{2}$ from the plots of the Stokes shifts against solvent polarity functions were found to be 0.78 and 0.79 for

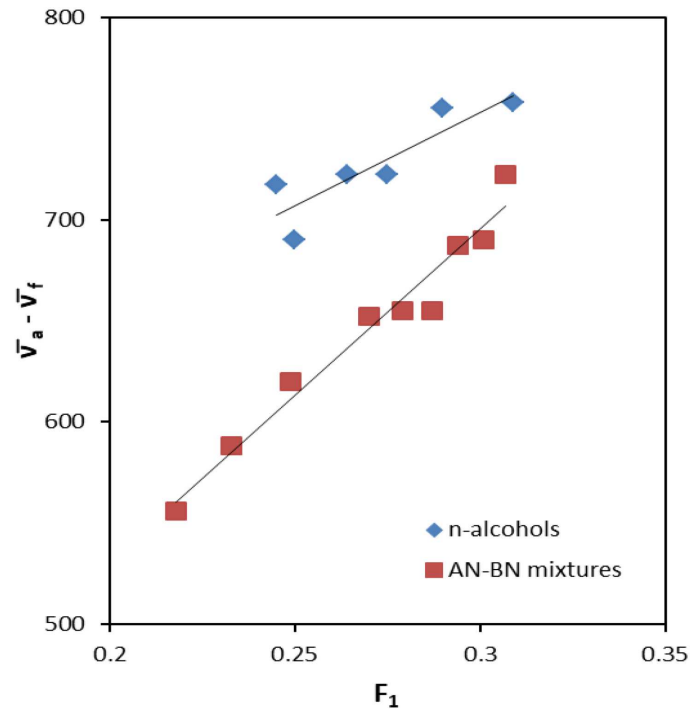

Fig. 4. Lippert's plot between the Stokes shift and solvent polarity function $F_{1}$.

Lippert's and Bakhshiev's equations in n-alcohols, respectively. It was seen that the alcoholic solvents caused deviations from Lippert's and Bakhshiev's equations $[4,20]$.

Lippert's theory assumes that general solvent effects are present in the solvent medium and the polarizability of the solute molecule is neglected. This model does not contain any chemical interactions. The direction of ground- and excited-dipole moments is parallel to each other, namely it is collinear. Deviations from Lippert's theory occur due to specific solute-solvent interactions such as hydrogen bonding or a formation of charge transfer states [7, 21]. 


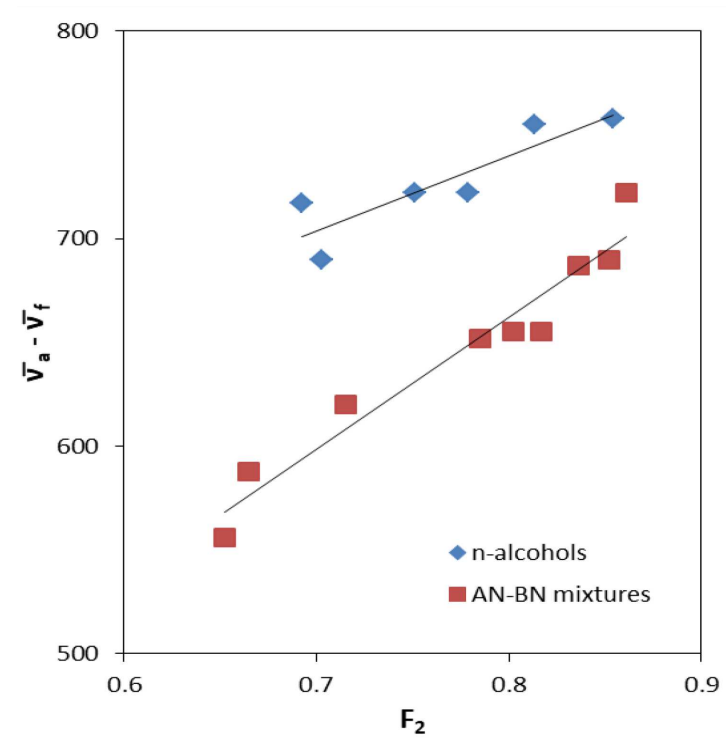

Fig. 5. Bakhshiev's plot between the Stokes shift and solvent polarity function $F_{2}$.

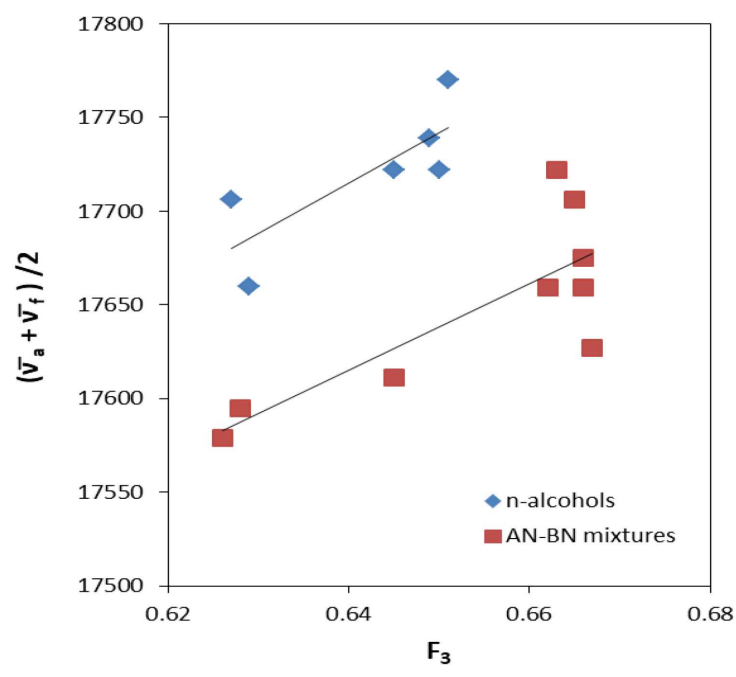

Fig. 6. Chamma-Viallet's plot between the Stokes shift and solvent polarity function $F_{3}$.

Bakhshiev's theory takes into account the solute polarizability besides specific solute-solvent interactions [7]. The direction of dipole moments of ground- and excited-dipole state is not collinear, but the linearity of the dipoles is close to each other. Deviations from the linearity of Bakhshiev's equation may result from an incomplete solvent relaxation prior to fluorescence emission or specific solute-solvent interactions, such as hydrogen bonding [5].

Chamma-Viallet's theory assumes that the molecular aspects of solvation are ignored in addition to Bakhshiev's assumptions. Also, in the case of Chamma-Viallet's method, the values of the excited-state dipole moment lie between those of Lippert's and Bakhshiev's [22]. In our study, it was seen that Chamma-Viallet's equation did not show any harmony with these interactions and therefore the excited-state dipole moments could not be determined.

Herein, the low $r^{2}$ values of 0.78 and 0.79 obtained from Lippert's and Bakhshiev's equations probably result from the formation of hydrogen bonding in n-alcohols. This hydrogen bonding occurs from the specific interactions which occur along with dipole-dipole interactions with the hydroxyl groups of protic n-alcohol solvents and the positively charged amino groups of PyB [23]. The formation of hydrogen bonding is probably under the influence of the hydrogen bond donor (HBD) acidity in protic n-alcohol solvents [24]. The $\alpha$ scale of solvent HBD acidities describes the ability of the solvent to donate a proton in a solvent-to-solute hydrogen bond [24]. Also, the values of the $\alpha$ scale for the alcohols between methanol and n-hexanol have been presented as ranging from 0.93 to 0.78 by Kamlet et al. [24]. Therefore, specific solutesolvent interactions occur as a result of the HBD effect in n-alcohol solvents. This $\alpha$ value of alcohols indicates that solvent HBD acidities play a role in solvent-soluble interactions and in non-HBD solvents $\alpha=0$ [24].

As Fig. 1 shows, on the other hand, pyronin B is an organic salt (containing $\mathrm{FeCl}_{4}^{-}$anion) which in polar solvents may affect the molecular structure and influence the electrostatic interactions with the solvent. At the same time, this may actually be the reason behind the small solvatochromism of the spectra in solvents. In this study, solvatochromism of PyB absorption as well as fluorescence spectra is really very small: $4 \mathrm{~nm}$ in n-alcohols and $6 \mathrm{~nm}$ in AN-BN mixtures for absorption, $3 \mathrm{~nm}$ in n-alcohols and $2 \mathrm{~nm}$ in $\mathrm{AN}-\mathrm{BN}$ mixtures for emission spectra.

Furthermore, as seen in Figs. 2 and 3, the spectra of the absorption and fluorescence at Jacobian form [25] have peaks which are changed from $2.146 \mathrm{eV}$ to $2.131 \mathrm{eV}$ for absorption and from $2.057 \mathrm{eV}$ to $2.046 \mathrm{eV}$ for fluorescence emission in n-alcohols, with increasing solvent polarity function. Likewise, these spectra have peaks which are changed from $2.146 \mathrm{eV}$ to $2.131 \mathrm{eV}$ for absorption and from $2.057 \mathrm{eV}$ to $2.046 \mathrm{eV}$ for emission in the mixtures, with increasing solvent polarity function. It is seen that the energy difference between the spectra is $0.015 \mathrm{eV}$ for absorption and $0.011 \mathrm{eV}$ for fluorescence emission in n-alcohols. In $\mathrm{AN}$ and $\mathrm{AN}-\mathrm{BN}$ solvent mixtures, the energy difference is $0.027 \mathrm{eV}$ for absorption and $0.007 \mathrm{eV}$ for fluorescence emission. These small energy values probably indicate that a charge transfer could not occur in the excited-state and therefore, the specific solutesolvent interactions may be considered to play a role in the occurrence of the energetic states.

The values of $r^{2}$ from Lippert's and Bakhshiev's equations - by eliminating the alcoholic solvents were found to be 0.96 and 0.94 , respectively in AN and $\mathrm{AN}-\mathrm{BN}$ solvent mixtures. These high values 


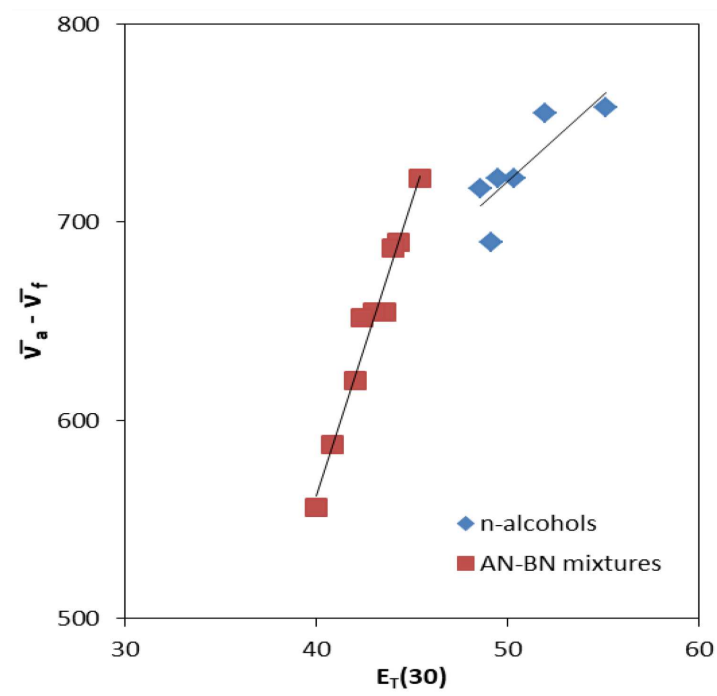

Fig. 7. The plots between the Stokes shift and solvent polarity parameter $E_{T}(30)$.

of $r^{2}$ indicate the general solvent effects. A similar situation has also been obtained for the interactions between fluorescein dye and $\mathrm{AN}$ and $\mathrm{AN}-\mathrm{BN}$ solvent mixtures [4].

It would also be useful to use the $E_{T}(30)$ function, the empirical measure of solvent polarity, to better understand the effects of polarization and hydrogen bonding on spectral properties [26]. Therefore, the plots of the Stokes shift $\left(\bar{\nu}_{a}-\bar{\nu}_{f}\right)$ against the values of solvent polarity parameter, $E_{T}(30)$ were drawn (see Fig. 7). From the linear regression analyses of these plots, $r^{2}$ values were found to be 0.7016 and 0.9716 for $n$-alcohols and the mixtures, respectively. If the dipole moment of a solute molecule is dependent only on solvent polarity, the plot of the $\left(\bar{\nu}_{a}-\bar{\nu}_{f}\right)$ versus $E_{T}(30)$ must have a linear relationship in the solvents [4]. Herein, while a satisfying fit was not observed for protic n-alcohols $\left(r^{2}=0.7016\right)$, a good fit was obtained with $r^{2}=0.9716$ for aprotic AN and AN-BN solvent mixtures. These findings are consistent with the results obtained from Lippert's and Bakhshiev's equations. Similar results have also been reported for fluorescein in n-acohols and $\mathrm{AN}$ and $\mathrm{AN}-\mathrm{BN}$ solvent mixtures [4].

\section{Conclusion}

The ground- and excited-state dipole moments of pyronin $\mathrm{B}$ (PyB) using the solvatochromic method and quantum-chemical calculations were determined in n-alcohols, acetonitrile (AN) and acetonitrile-benzene (AN-BN) solvent mixtures. The ground-state dipole moments were calculated using both the experimental Guggenheim-Smith method (GSM) and the theoretical (SCF MO $\mathrm{LCAO}$ ) in CNDO/2 and MNDO methods. The values of the theoretical dipole moments of $\mathrm{PyB}$ were found to be $11.29 \mathrm{D}$ for $\mathrm{CNDO} / 2$ and $3.43 \mathrm{D}$ for
MNDO methods, respectively. The experimental ground-state dipole moment values increased from $3.13 \mathrm{D}$ to $13.77 \mathrm{D}$ with decreasing polarity from methanol to n-hexanol in n-alcohols and from 9.25 to 14.63 with decreasing polarity in $\mathrm{AN}$ and $\mathrm{AN}-$ BN solvent mixtures. The excited-state dipole moments were determined from the plots of the Stokes shifts against solvent polarity functions by means of Lippert's and Bakhshiev's equations in n-alcohols and AN and AN-BN solvent mixtures. The excitedstate dipole moments of PyB were lower than those of the ground-state in n-alcohols and the mixtures. This indicated that the ground-state was more polar than the ground-state. Moreover, it was determined that the ground-state compared to the excited-state was more stable. The specific solute-solvent interactions were estimated to be due to hydrogen bonding between PyB and n-alcohol molecules. It was seen that the low energetic states occurred via the specific solute-solvent interactions instead of the charge transfer. The general solvent effects were seen in $\mathrm{AN}$ and $\mathrm{AN}-\mathrm{BN}$ solvent mixtures. All of these interactions were confirmed by means of Lippert's and Bakhshiev's equations and the use of the solvent polarity parameter $E_{T}(30)$.

\section{References}

[1] N. Tewari, N.K. Josh, R. Rautela, R. Gahlaut, H.C. Joshi, S. Pant, J. Mol. Liq. 160, 150 (2011).

[2] L. Ziółek, K. Palewska, J. Lipiński, J. Sworakowski, S. Nescpůrek, S. Böhm, E.C. Meister, Acta Phys. Pol. A 88, 283 (1995).

[3] A. Al Sabahi, S.N. Al Busafi, F.O. Suliman, S.M. Al Kindy, J. Mol. Liq. 307, 112967 (2020).

[4] B. Acemioğlu, M. Arık, H. Efeoğlu, Y. Onganer, J. Mol. Struct. (Theochem.) 548, 165 (2001).

[5] C. Parkanyi, C. Boniface, J.-J. Aaron, M.D. Gaye, L. von Szentpaly, R. Ghosh, K.S. RaghuVeer, Struct. Chem. 3, 277 (1992).

[6] G.V. Muddapur, V.V. Koppal, N.R. Patil, R.M. Melavanki, AIP Conf. Proc. 1728, 020373-1 (2016).

[7] M. Jozefowicz, P. Milart, J.R. Heldt, Spectrochim. Acta Part A 74, 959 (2009).

[8] J.J.P. Stewart, J. Computer-Aided Mol. Des. 4, 1 (1990).

[9] F. Moll, E. Lippert, Z. Elektrochem. 58, 853 (1954).

[10] B. Acemioglu, M. Arık, Y. Onganer, J. Lumin. 97, 153 (2002).

[11] D.L. Andrews, Laser in Chemistry, Springer-Verlag, Berlin 1990. 
[12] M.R. Rostami, M. Kaya, B. Gür, Y. Onganer, K. Meral, Appl. Surf. Sci. 359, 897 (2015).

[13] E. Lippert, Z. Electrochem. 61, 962 (1957).

[14] N.G. Bakhshiev, Opt. Spektrosk. 16, 821 (1964).

[15] A. Chamma, P. Viallet, C.R. Acad. Sci. Paris Ser. C 270, 1901 (1970).

[16] P. Suppan, Chem. Phys. Lett. 94, 272 (1983).

[17] D.R. Besirre, E.L. Quitevis, J. Phys. Chem. 98, 13083 (1994).

[18] J.J. Aaron, M.D. Gaye, C. Parkanyi, N.S. Cho, L.V. Szentpaly, J. Mol. Struct. 156, 119 (1987).

[19] C. Reichardt, Solvents and Solvent Effects in Organic Chemistry, VCH, Weinheim 1990 .

[20] S. Arathi Rani, J. Sobhanadri, T.A. Prasada Rao, Spectrochim. Acta Part A 51, 2473 (1995).
[21] J.R. Lakowicz, Principle of Fluorescence Spectroscopy, Plenum Press, New York 1983.

[22] J. Thipperudrappa, D.S. Biradar, S.R. Manohara, S.M. Hanagodimath, S.R. Inamadar, R.J. Manekutla, Spectrochim. Acta Part A 69, 991 (2008).

[23] Y. Onganer, E.L. Quitevis, J. Phys. Chem. 96, 7996 (1992).

[24] M.J. Kamlet, J.-L.M. Abboud, M.H. Abraham, R.W. Taft, J. Org. Chem. 48, 2877 (1983).

[25] J. Mooney, P. Kambhampati, J. Phys. Chem. Lett. 4, 3316 (2013).

[26] K. Chandrasekhar, L.R. Naik, H.M. Sureh Kumar, N.N. Math, Indian J. Pure Appl. Phys. 44, 292 (2006). 